\title{
Compact Wake-Up Module Design Based on an Energy-Harvesting Rectenna for Wireless Sensor Receivers
}

\author{
Sang-Min Han, ${ }^{1}$ Yongwan Lee, ${ }^{1}$ Taemin Choi, ${ }^{1}$ Seok-Jae Lee, ${ }^{1}$ Jung-Woo Baik, \\ Jongsik Lim, ${ }^{1}$ and Dal Ahn ${ }^{1}$ \\ ${ }^{1}$ Soonchunhyang University, Asan, Chungnam 336-745, Republic of Korea \\ ${ }^{2}$ Convergence R\&D Center, Samsung S1 Corporation, Seoul 100-773, Republic of Korea
}

Correspondence should be addressed to Dal Ahn; dahnkr@sch.ac.kr

Received 1 December 2014; Revised 13 February 2015; Accepted 16 February 2015

Academic Editor: Giuseppe Castaldi

Copyright (C) 2015 Sang-Min Han et al. This is an open access article distributed under the Creative Commons Attribution License, which permits unrestricted use, distribution, and reproduction in any medium, provided the original work is properly cited.

A new compact energy-harvesting module is proposed with compact design techniques. The rectifying circuit eliminates the bandpass filter and matching circuit, based on an active antenna concept and a direct matching technique. For exact circuit impedance, via holes are processed with precise fabrication techniques. The implemented circuit has achieved a circuit size reduction of $76.7 \%$. The proposed system has been applied to a wireless wake-up receiver system with excellent operating performance.

\section{Introduction}

Since the Space Power Satellite (SPS) project launched from NASA in the US in 1960s, various types of wireless power transfer technologies have been developed $[1,2]$. While the rectifying antenna (rectenna) system can convert from microwave signal to DC voltage for a long distance energy transfer, the electromagnetic resonance type power transfer delivers low frequency energy for a short distance of a few meters. Due to less power harvested by rectenna systems, for last ten years, commercial interests have moved to the electromagnetic resonance type power transfer that can deliver a few watts $[3,4]$. Even though several innovative approaches have been studied to improve rectenna performance, the transmission loss for long distance makes low total transfer efficiency. However, the electromagnetic resonance type system has a limitation of transmission distance. Recently, Japan has developed a new commercial SPS with 5 billion tiny rectennas that is capable of producing more than $1 \mathrm{GW}$. It is considered that the higher solar power and continuous sunny sky in space can compensate for the low transfer efficiency [5]. In addition, this is the only system that can transfer electromagnetic energy by microwave propagation for a long distance. Moreover, the rectenna system can be applied to wireless system activation and energy harvesting from versatile wireless power resources [6-10].

In this paper, a new compact design of a rectenna system is proposed for wireless sensor receiver applications. Because the rectenna applications have developed towards being mounted on electric devices to supply electric power, compact and planar designs are required to integrate with a small form factor and lesser number of devices. In particular, as a wake-up module is an auxiliary device for low-power sensor receiver systems, it is required to be planar and more compact implementation. Therefore, in order to reduce the number of components, the Active Integrated Antenna (AIA) technology has been applied [9-11]. Additionally, a direct matching technique is proposed for simple and efficient circuit design [12-14]. By eliminating the Band-Pass Filter (BPF) and impedance matching circuit, rectifying circuits can be simplified for integration. Furthermore, a precise fabrication technique has been introduced for coaxial feeding and impedance matching processes. This paper is organized as follows. Following a brief introduction, a compact rectenna design is described in Section 2. Section 3 explains the experimental results for performance evaluations and wireless system applications. Finally, Section 4 provides the conclusion and discusses applications and commercial feasibility. 


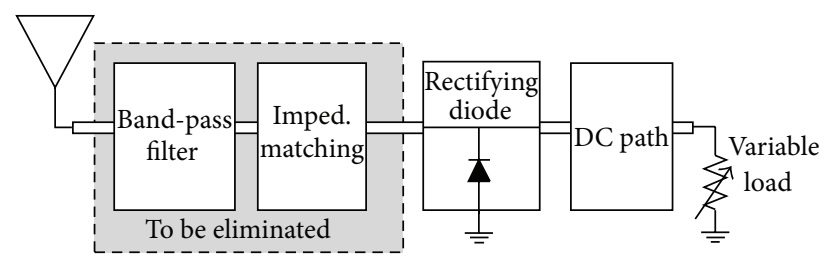

FIGURE 1: Design concept for the compact rectenna system design.

\section{Compact Rectenna Design Using a Circular Sector Antenna}

In this section, a compact rectenna design technology is proposed based on the AIA concept and the direct matching technique. The AIA system is designed with a radiating element playing additional roles of front-end circuits. Because an energy-harvesting system requires a small form factor to be integrated into an energy-hungry system, a compact design technology needs to be composed of a lesser number of components. Figure 1 describes the system diagram of the proposed rectenna system design. The conventional rectenna consists of a receiving antenna, a BPF for harmonic rejection, an impedance matching circuit, a rectifying diode, and a Low Pass Filter (LPF) for a DC path. A received RF/microwave signal passes the BPF and then is rectified by the Schottky diode. Because the receiving signal at a fundamental frequency component should be inserted into the diode, the impedance matching circuit is essential for efficient system performance. Moreover, the BPF needs to be terminated at $50 \mathrm{Ohm}$. DC and harmonic frequency components containing analog energy are generated at the diode. To increase the conversion efficiency, the harmonics should be blocked and reflected into the diode by filters, such as the BPF and LPF. The reflected harmonics can regenerate more DC energy. The proposed system has achieved compactness by eliminating two circuit components. While the BPF can be removed by the AIA concept, the matching circuit can be omitted by the direct matching technique.

2.1. Circular Sector Antenna Design. In this paper, a circular sector antenna is utilized with harmonic-rejecting characteristics that can play the role of the BPF. The circular sector antenna has 120-degree hatched sector. The harmonicrejecting characteristics can be achieved by feeding along the 30 -degree line from the edge of a sector [10]. The resonant frequency is determined by the radius of a circular patch. The antenna is implemented on an RT/Duroid 5880 substrate with a dielectric constant of 2.2 and thickness of 31 mils. The radius is $19.8 \mathrm{~mm}$, and the feeding point is $2.79 \mathrm{~mm}$ distant from the center for $50 \Omega$ input impedance. In Figure 2(a), the measured return loss presented a center frequency of $2.505 \mathrm{GHz}$ and a bandwidth of $30 \mathrm{MHz}$ for $50 \Omega$ matched coaxial feeding; however, no resonances were evident at the 2nd and 3rd harmonic frequencies of about $5.0 \mathrm{GHz}$ and $7.5 \mathrm{GHz}$. The shifted harmonic frequencies appeared around $4 \mathrm{GHz}$ and $6 \mathrm{GHz}$. Therefore, the circular sector antenna can play the additional role of a BPF to block the reradiation of harmonic frequency components that are generated at a nonlinear diode [10]. The measured radiation patterns are depicted in Figure 2(b). The radiation patterns of the antenna are typical ones of a patch antenna. The gain of this antenna is found to be $6.33 \mathrm{dBi}$. It presents linear polarization with an $E$-plane along the $x$-axis direction in Figure 3(a). Although its feeding point is near the center, the antenna has linear polarization and low cross polarization level of less than $20 \mathrm{~dB}$.

Secondly, a direct matching technique is applied by searching the variable input impedance of the circular sector antenna. Because the antenna can keep harmonic-rejecting characteristics while it is fed at any point along the 30-degree line, the feeding points are selected along the line. From Figure 3(a), the marks from " $a$ " to " $e$ " in the $x$-direction are located at a distance of $1,2,3,4$, and $5 \mathrm{~mm}$ apart from the center of the antenna. The input impedance trace is investigated, as shown in Figure 3(b). From the measured results, the impedance is linearly moved and monotonically decreased. The optimal matching point can be found for the variable impedance.

2.2. Compact Rectifying Circuit Design. The compact rectifying circuit is designed for wireless wake-up modules. Figure 4 shows a schematic diagram of a rectifying circuit consisting of a single diode and a four-stage LPF, which could be simply implemented, thanks to the proposed minimizing techniques. The Schottky diode (HSMS-2850, Avago Technologies) is utilized for high speed microwave switching [15]. The four-stage LPF has lumped components of $L 1=4.7 \mathrm{nH}$, $C 1=2.7 \mathrm{pF}, L 2=5.6 \mathrm{nH}$, and $C 2=2.2 \mathrm{pF}$. As the input impedance of the rectifying circuit is dependent on the value of a load resistance, the experiments have been conducted for various loads. The measured input impedance at $2.483 \mathrm{GHz}$ for real and imaginary values is presented in Figure 5.

From the measured input impedance of the rectifying circuit, the optimum feeding point of the circular sector antenna was searched for efficient impedance matching. As the impedance of a rectifying circuit is variable for load resistance, the impedance is searched for approximate values of antenna input impedance. And then, for a fixed load resistance, a matched antenna feeding point is investigated by a simulator. In this paper, from the input impedance trace in Figure 3(b), a matched point was found near point " $c$ " for $300 \Omega$ load resistance. For minor tuning of imaginary impedance, the final feeding point was slightly adjusted on the $y$-axis.

2.3. Implementation of the Compact Rectenna System. The rectenna system for an energy-harvesting system module was implemented on both sides of a PCB, by back-to-back integration. In order to keep the same feeding condition for sensitive input impedance of the antenna, the insides of coaxial via holes were coated with copper for every prototype. Figure 6(a) shows a cross-section of the rectenna configuration. The rectifying circuit was implemented on the backside of the patch antenna, fed through one coaxial feeder. A ground plane was realized only on the antenna substrate, 


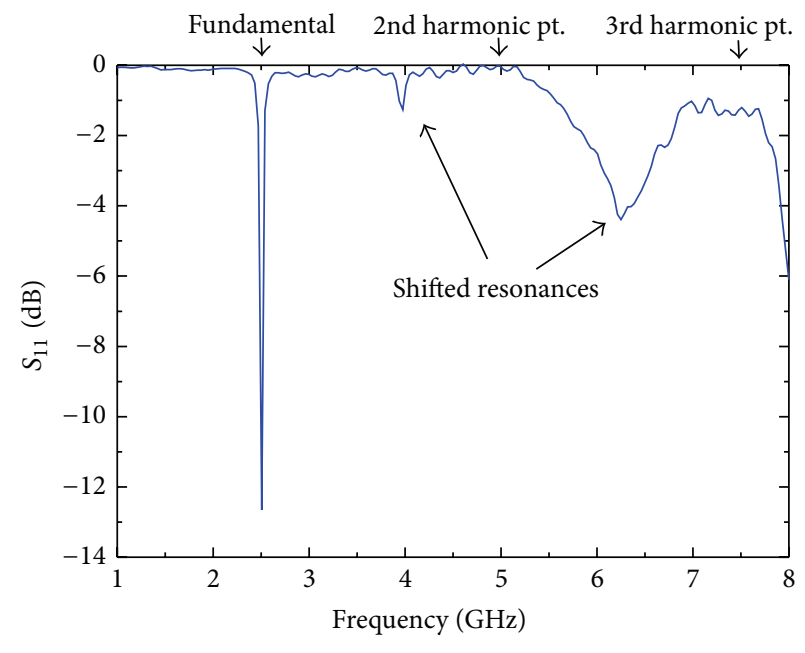

(a)

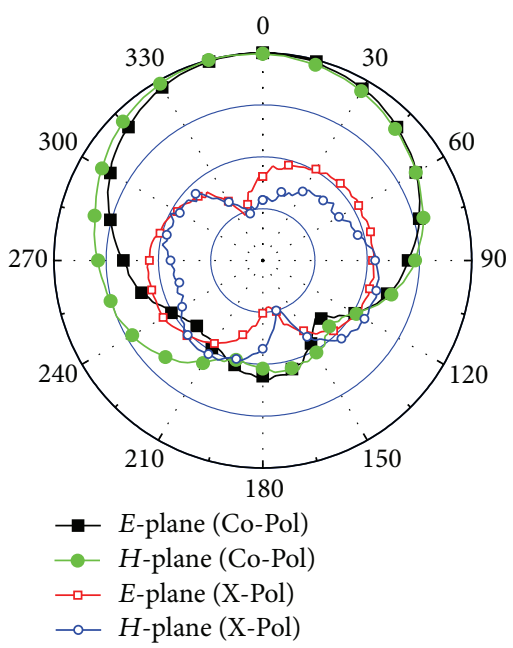

(b)

FiguRE 2: Characteristics of the circular sector antenna. (a) $S_{11}$. (b) Radiation patterns (10 dB/div.).

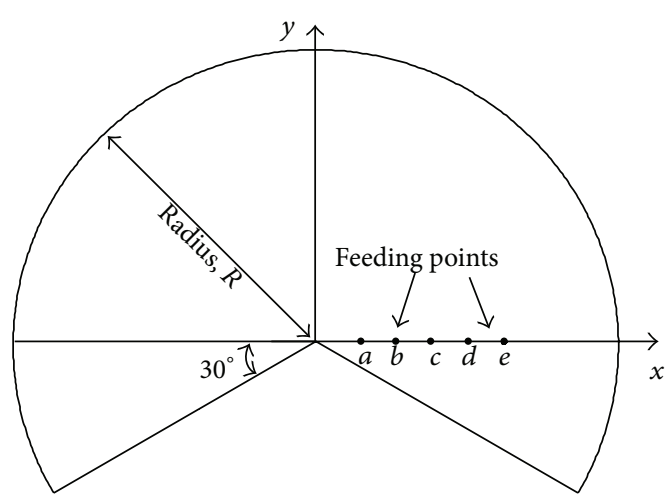

(a)

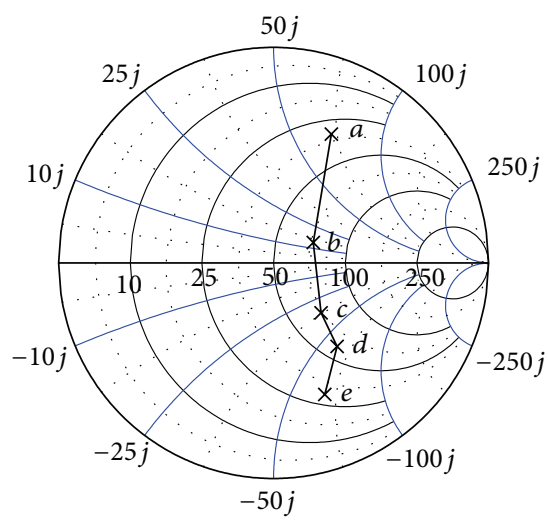

(b)

FIGURE 3: Coaxial-fed circular sector antenna. (a) Layout with variable feeding points. (b) Input impedance trace along feeding points.

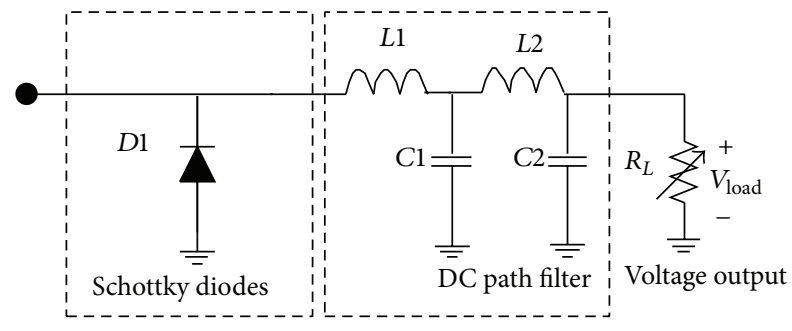

FIGURE 4: Schematic diagram of the rectifying circuit.

which makes two circuit boards share the common ground plane. Figure 6(b) describes the via-hole configuration. After screwing a hole, the inner surface was coated by conductor material that is the same as that of via holes. As the process can improve the contact with via holes, this makes it possible to take reliable and precise implementation. In addition, insulating ring bands were mounted in the vicinity of via

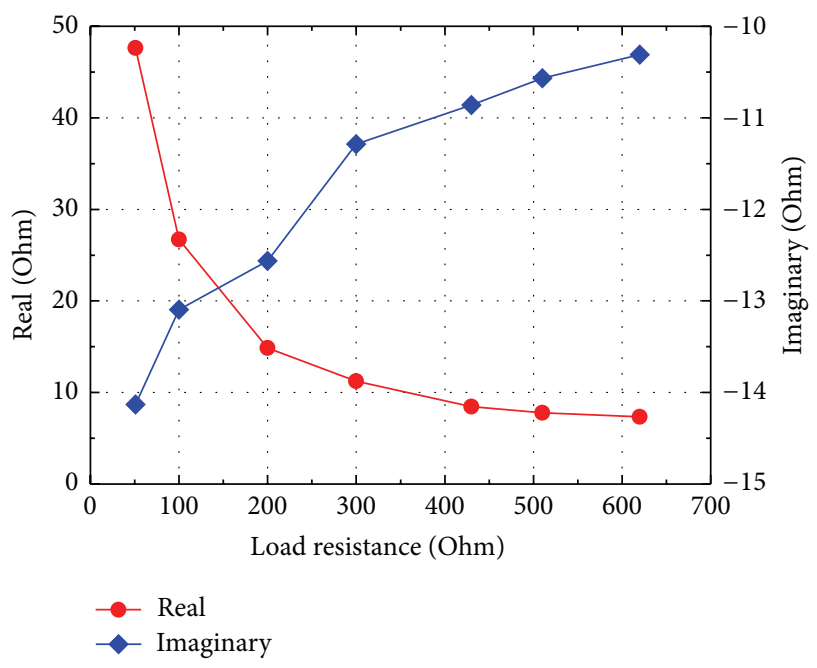

FIGURE 5: Measured input impedance of the compact rectifying circuit for variable load resistance. 


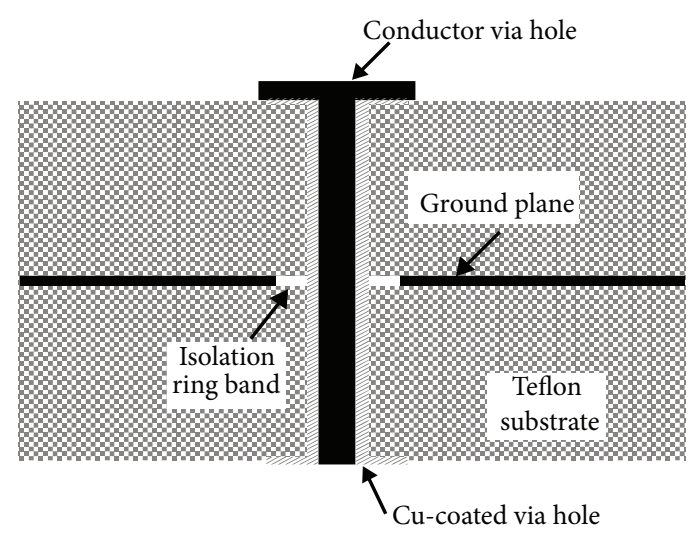

(a)

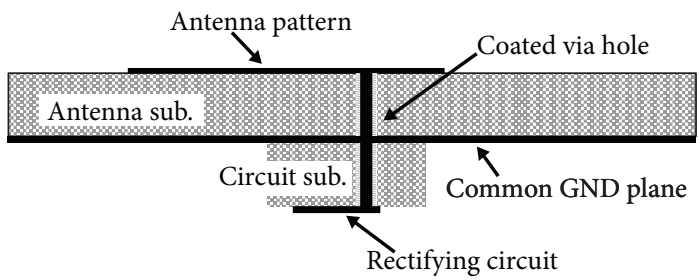

(b)

FIGURE 6: Cross-sections of (a) the fabricated rectenna with a direct matching technique and (b) the via hole.

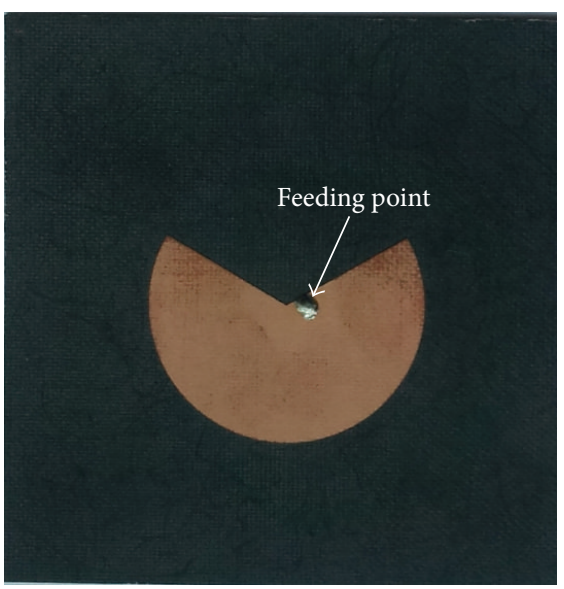

(a)

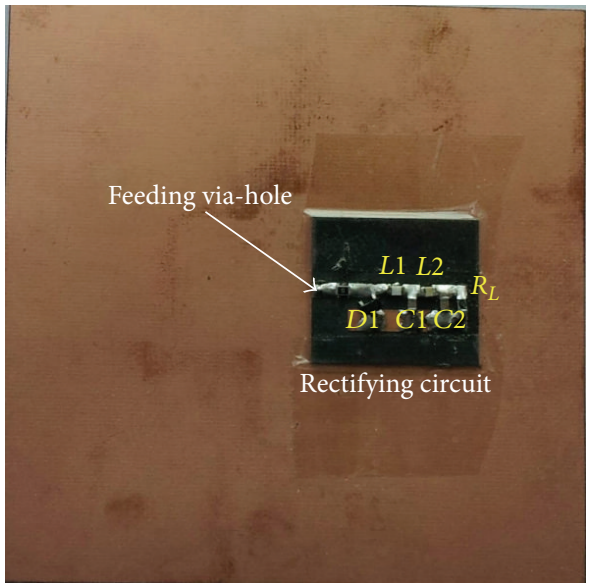

(b)

FIGURE 7: Photographs of the implemented compact rectenna module. (a) Front side. (b) Rear side.

holes for electrical isolation. Photographs of the fabricated energy-harvesting rectenna system are shown in Figure 7. The system size is $19.8 \times 19.8 \times 1.6 \mathrm{~mm}^{3}$, which can achieve a form factor reduction of $76.7 \%$, compared with previous designs $[9,10]$.

\section{Experiments for Performance Evaluations and Application to a Wake-Up Receiver System}

In this section, the proposed compact energy-harvesting module was evaluated and applied to an electric power supplier for an energy-hungry system. A wireless communication receiver system cooperated with the compact rectenna module. The rectified DC voltage was provided to wake up the receiver for low-power sensor network applications.

In Figure 8, a measurement setup is configured to evaluate the energy conversion performance from microwave power to DC, of conversion efficiency and conversion voltages. A microwave signal is generated at $2.483 \mathrm{GHz}$ and amplified from a drive amplifier and a power amplifier for sufficient radiating power. A receiving circular sector antenna at a distance $d$ apart from a transmitting antenna receives the Continuous Wave (CW). The received signal is rectified by the proposed compact rectenna module. The wireless radiating site is surrounded by electromagnetic absorbing materials. The distance $d=0.5 \mathrm{~m}$ is satisfied with a farfield condition of more than $0.21 \mathrm{~m}$. As received power was measured by a microwave power meter using the same circular sector antenna as that of the module at the same position, the converted DC power was measured by a voltage meter. To achieve maximum power transfer, the circular sector antenna is aligned at the copolarization with a Tx. horn antenna.

Figure 9 presents the measured conversion efficiency and conversion voltages of the proposed compact rectenna module. The experiments were performed for various load resistances from $51 \Omega$ to $620 \Omega$. The measured efficiencies increased for receiving power up to $65 \%$ at $200 \Omega$ and $300 \Omega$. The output voltages were converted up to $1.57 \mathrm{~V}$ at a load of $620 \Omega$. The experimental results of energy conversion show 


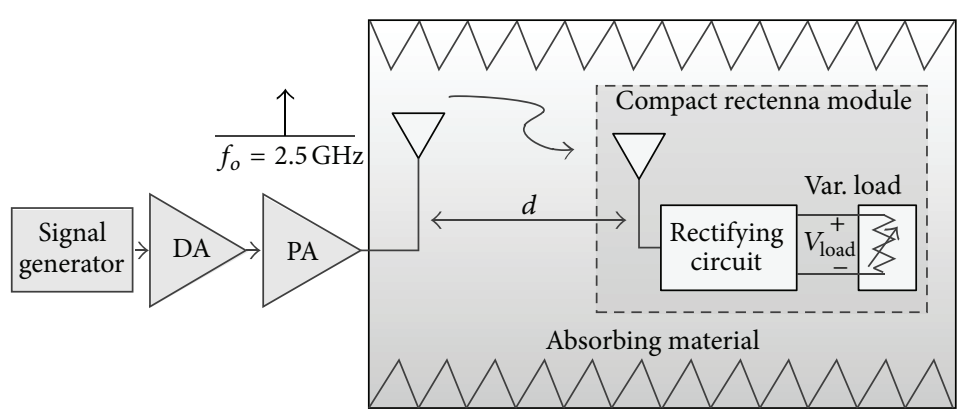

FIGURE 8: Measurement setup for performance evaluation of the rectenna module.

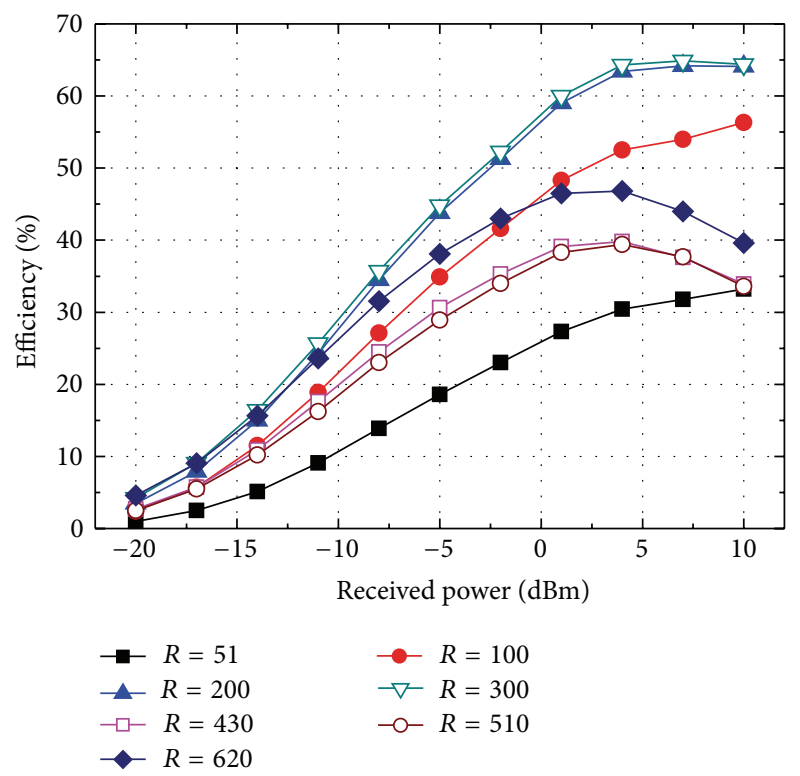

(a)

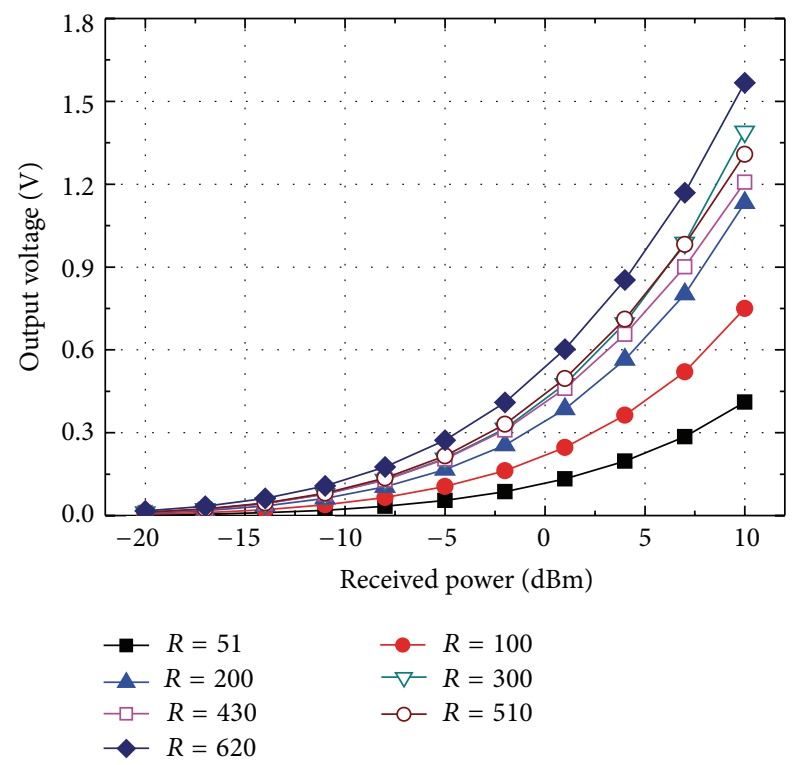

(b)

FIGURE 9: Measured results of the proposed compact energy-harvesting module. (a) Conversion efficiencies. (b) Conversion voltages.

excellent performances for direct matching, compared to one with a conventional matching circuit [16].

The proposed energy-harvesting module was applied to a receiver system for wireless sensor receiver systems. Each transceiver was designed for random digital data transmission. The On-Off Keying (OOK) modulated signal was excited with a data rate of $2 \mathrm{Mbps}$ at a carrier frequency of $2.483 \mathrm{GHz}$. The receiver system consists of two-stage Low Noise Amplifiers (LNAs), an envelope detector, a LPF, and a comparator, which are power supplied through an analog switching circuit (FSA1156, Fairchild Semiconductor) from a power supply, as shown in Figure 10. The wake-up system is designed with a power supply control to achieve low-power consumption architecture for sensor receiver systems, instead of an RF path switching. The switching circuit is controlled by the proposed compact rectifying module, which is optimally designed for low voltage operation of less than $0.45 \mathrm{~V}$. A DCDC converter was utilized for stable switch operation. When the received microwave power comes to a wireless sensor, the rectenna module makes the receiver system awaken by switching "On". Therefore, the receiving power reaches a threshold, and the module wakes up the receiver system by providing DC power.

Figure 11 shows the experimental results of the wake-up receiver system. In order to check the proper operation of the receiver, two kinds of states were measured at the receiver system. For various received power levels, the LNA operation is presented for power gains. In Figure 11(a), the LNA starts operating and makes a power gain at the threshold received power of $-5 \mathrm{dBm}$. The received spectrum is presented for modulated signal. The demodulation performances are searched for OOK-modulated digital data. Figure 11(b) shows the recovered digital data for on/off receiver status ( $\mathrm{Rx}_{-} 1$ for off and $\mathrm{Rx}_{2} 2$ for on status). For woken-up state, the receiver system demodulated the digital data with excellent performance.

\section{Conclusion}

The new design method of a compact rectenna system is proposed for wireless sensor receiver system applications. The proposed rectifying system has achieved compactness and 


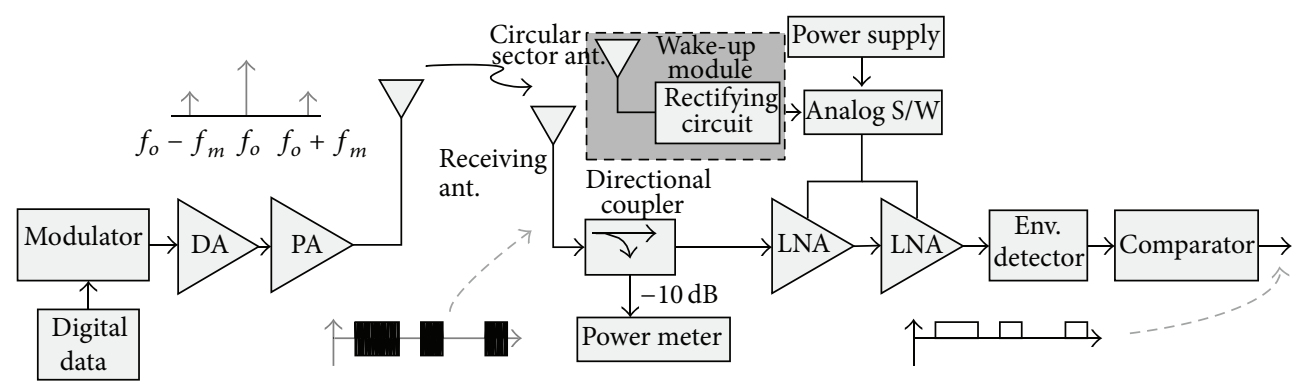

FIGURE 10: Sensor receiver system application of the compact rectenna module.

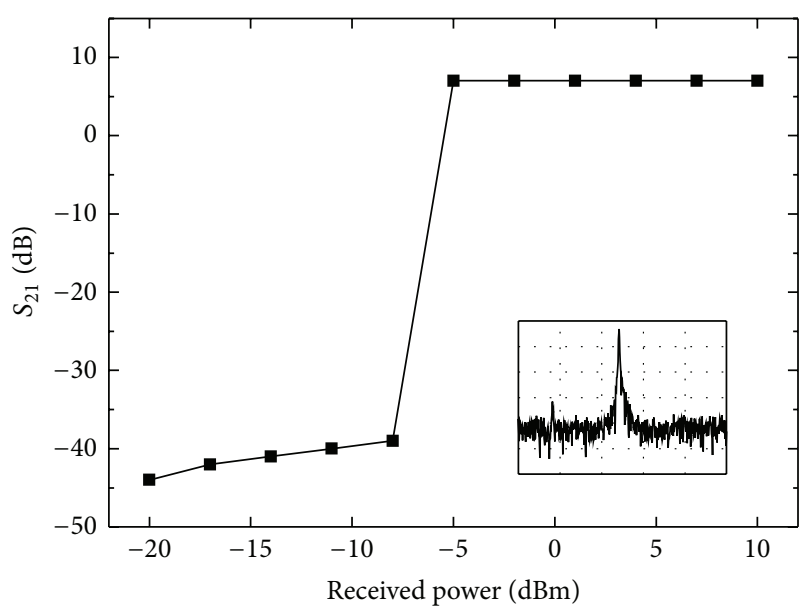

(a)
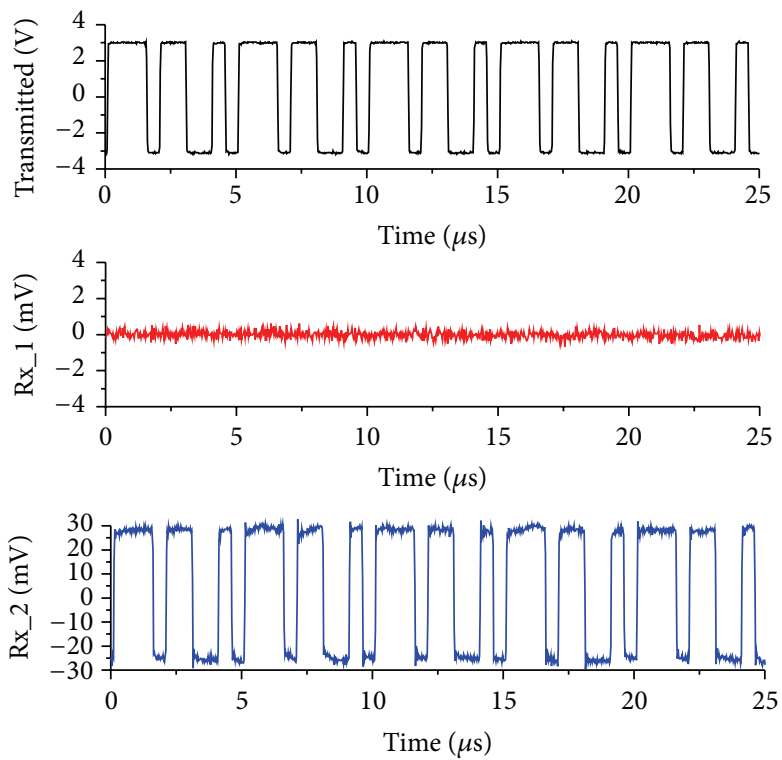

(b)

FIGURE 11: Wake-up performance evaluations for received RF power. (a) LNA operation. (b) Transmitted and recovered digital data.

a small form factor by eliminating the circuit components of BPF and impedance matching circuit. Two design technologies are applied with the active integrated antenna concept and the direct matching technique. For the exact experiments for input impedance, precise fabrication was processed for via holes. The proposed compact energy-harvesting module was applied to a wake-up wireless receiver system and evaluated with excellent operating performance. The proposed system can be an excellent candidate for various wireless devices to be activated and operated. Furthermore, it is expected for the module to be fabricated with much smaller form factor by the support of material and fabrication technologies. The proposed system module can be applicable to various wireless devices for sensor network applications.

\section{Conflict of Interests}

The authors declare that there is no conflict of interests regarding the publication of this paper.

\section{Acknowledgments}

This work was supported by the National Research Foundation of Korea (NRF) grant funded by the Korean government (Grant no. 2009-0065375) and the Soonchunhyang University Research Fund.

\section{References}

[1] W. C. Brown, "The history of power transmission by radio waves," IEEE Transactions on Microwave Theory and Techniques, vol. 32, no. 9, pp. 1230-1242, 1984.

[2] C. R. Valenta and G. D. Durgin, "Harvesting wireless power: survey of energy-harvester conversion efficiency in far-field, wireless power transfer systems," IEEE Microwave Magazine, vol. 15, no. 4, pp. 108-120, 2014.

[3] A. Kurs, A. Karalis, R. Moffatt, J. D. Joannopoulos, P. Fisher, and M. Soljačić, "Wireless power transfer via strongly coupled magnetic resonances," Science, vol. 317, no. 5834, pp. 83-86, 2007. 
[4] J.-H. Kim, B.-C. Park, and J.-H. Lee, "New analysis method for wireless power transfer system with multiple n resonators," Journal of Electromagnetic Engineering and Science, vol. 13, no. 3, pp. 173-177, 2013.

[5] S. Sasaki, "It's always sunny in space," IEEE Spectrum, vol. 51, no. 5, pp. 46-51, 2014.

[6] J. A. C. Theeuwes, H. J. Visser, M. C. V. van Beurden, and G. J. N. Doodeman, "Efficient, compact, wireless battery design," in Proceedings of the 10th European Conference on Wireless Technology (ECWT '07), pp. 233-236, October 2007.

[7] T. Choi and S.-M. Han, "Compact rectenna system design using a direct impedance matching method," Journal of Korean Institute of Electromagnetic Engineering and Science, vol. 24, no. 3, pp. 286-291, 2013.

[8] Z. Harouni, L. Cirio, L. Osman, A. Gharsallah, and O. Picon, "A dual circularly polarized $2.45-\mathrm{GHz}$ rectenna for wireless power transmission," IEEE Antennas and Wireless Propagation Letters, vol. 10, pp. 306-309, 2011.

[9] J.-Y. Park, S.-M. Han, and T. Itoh, "A rectenna design with harmonic-rejecting circular-sector antenna," IEEE Antennas and Wireless Propagation Letters, vol. 3, no. 1, pp. 52-54, 2004.

[10] S.-M. Han, J.-Y. Park, and T. Itoh, "A self-biased receiver system using the active integrated antenna," IEICE Transactions on Communications, vol. 89, no. 2, pp. 570-575, 2006.

[11] S. Lim, K. M. K. H. Leong, and T. Itoh, "Adaptive power controllable retrodirective array system for wireless sensor server applications," IEEE Transactions on Microwave Theory and Techniques, vol. 53, no. 12, pp. 3735-3743, 2005.

[12] A. S. Andrenko, Y. Ikeda, M. Nakayama, and O. Ishida, "Impedance matching in active integrated antenna receiver front end design," IEEE Microwave and Wireless Components Letters, vol. 10, no. 1, pp. 16-18, 2000.

[13] D.-J. Kim, Y.-M. Moon, S.-H. Park, Y.-E. Kim, and K.-S. Min, "A small monopole antenna with novel impedance matching structure," in Proceedings of the 36th European Microwave Conference, pp. 819-822, September 2006.

[14] W. Lin and Q.-X. Chu, "A novel RFID tag antenna for matching complex impedances on $915 \mathrm{MHz}$ and $2.45 \mathrm{GHz}$ bands," in Proceedings of the Asia-Pacific Microwave Conference (APMC '10), pp. 2248-2251, December 2010.

[15] J. Park, Y. Kim, Y. J. Yoon, J. So, and J. Shin, "Rectifier design using distributed Greinacher voltage multiplier for high frequency wireless power transmission," Journal of Electromagnetic Engineering and Science, vol. 14, no. 1, pp. 25-30, 2014.

[16] S.-M. Han, J.-Y. Park, and T. Itoh, "Active integrated antenna based rectenna using the circular sector antenna with harmonic rejection," in Proceedings of the IEEE Antennas and Propagation Society International Symposium, vol. 4, pp. 3533-3536, Monterey, Calif, USA, June 2004. 

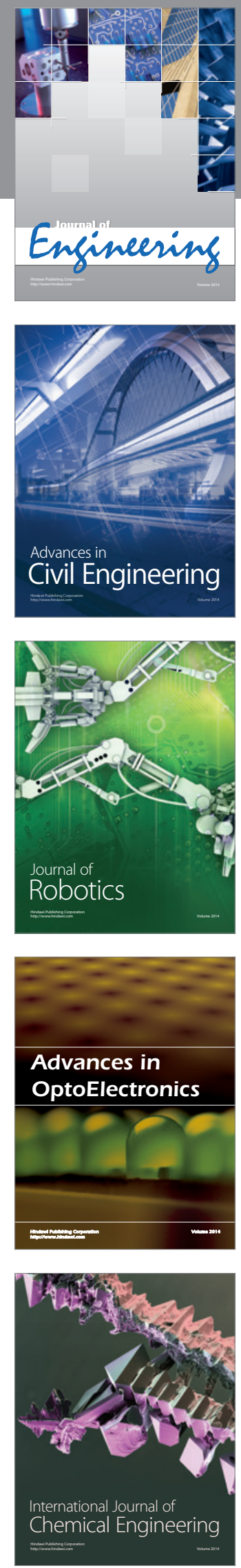

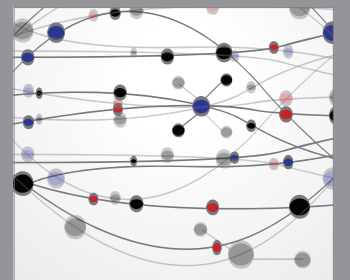

The Scientific World Journal
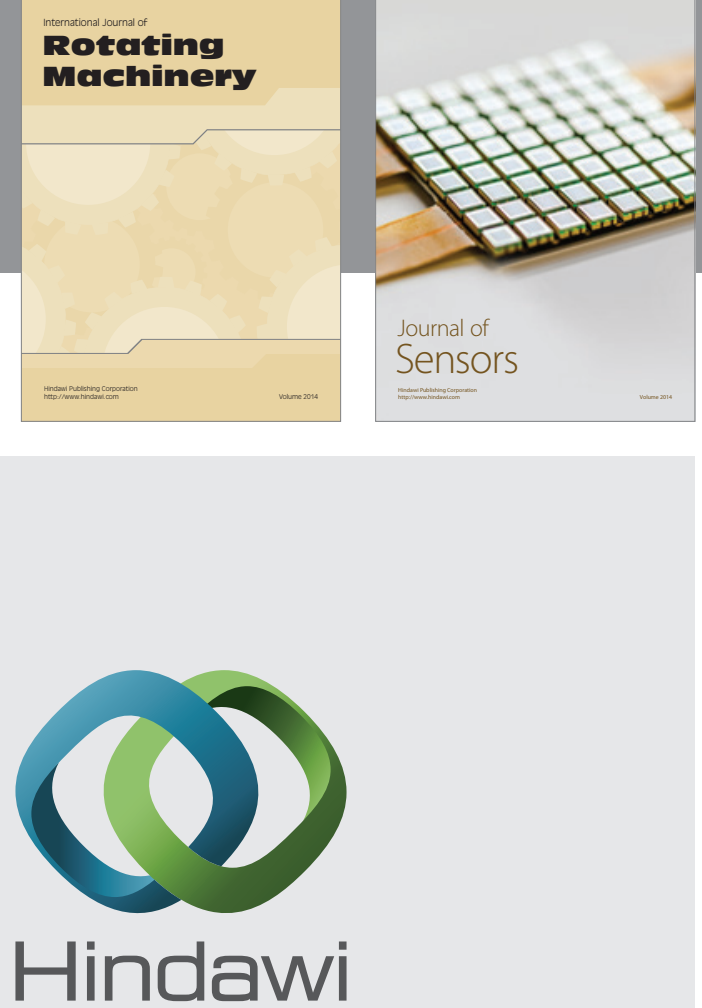

Submit your manuscripts at http://www.hindawi.com
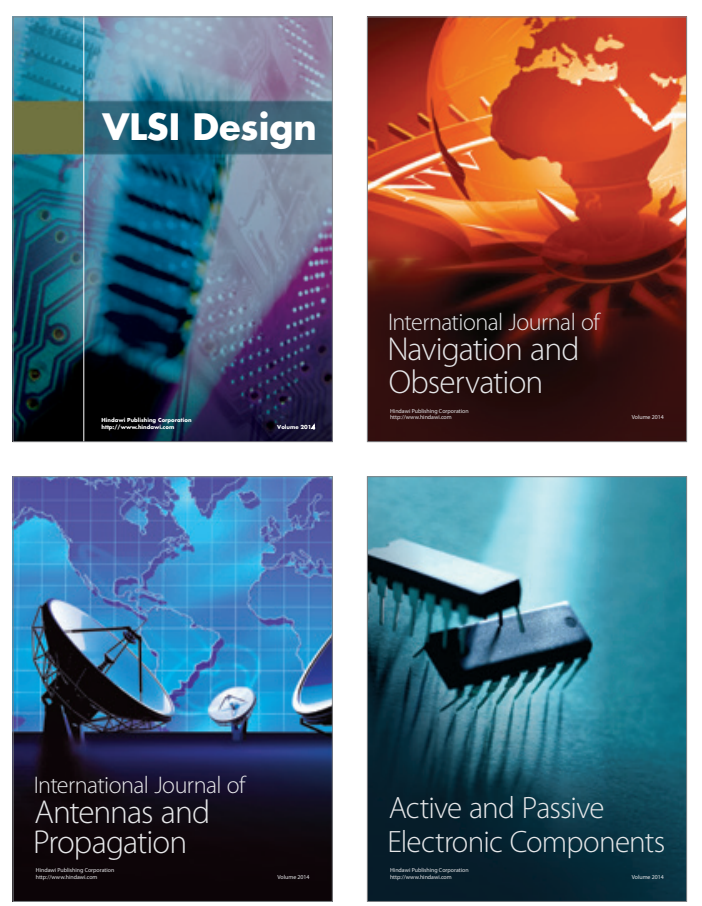
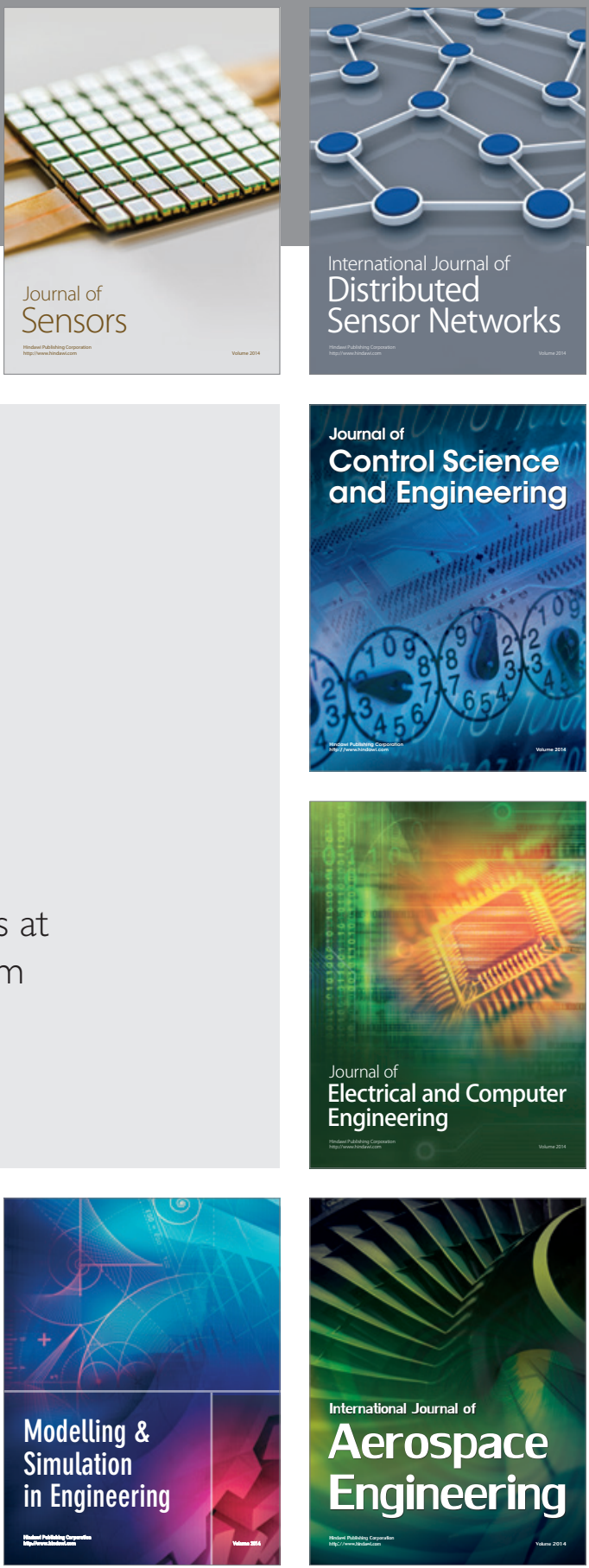

Journal of

Control Science

and Engineering
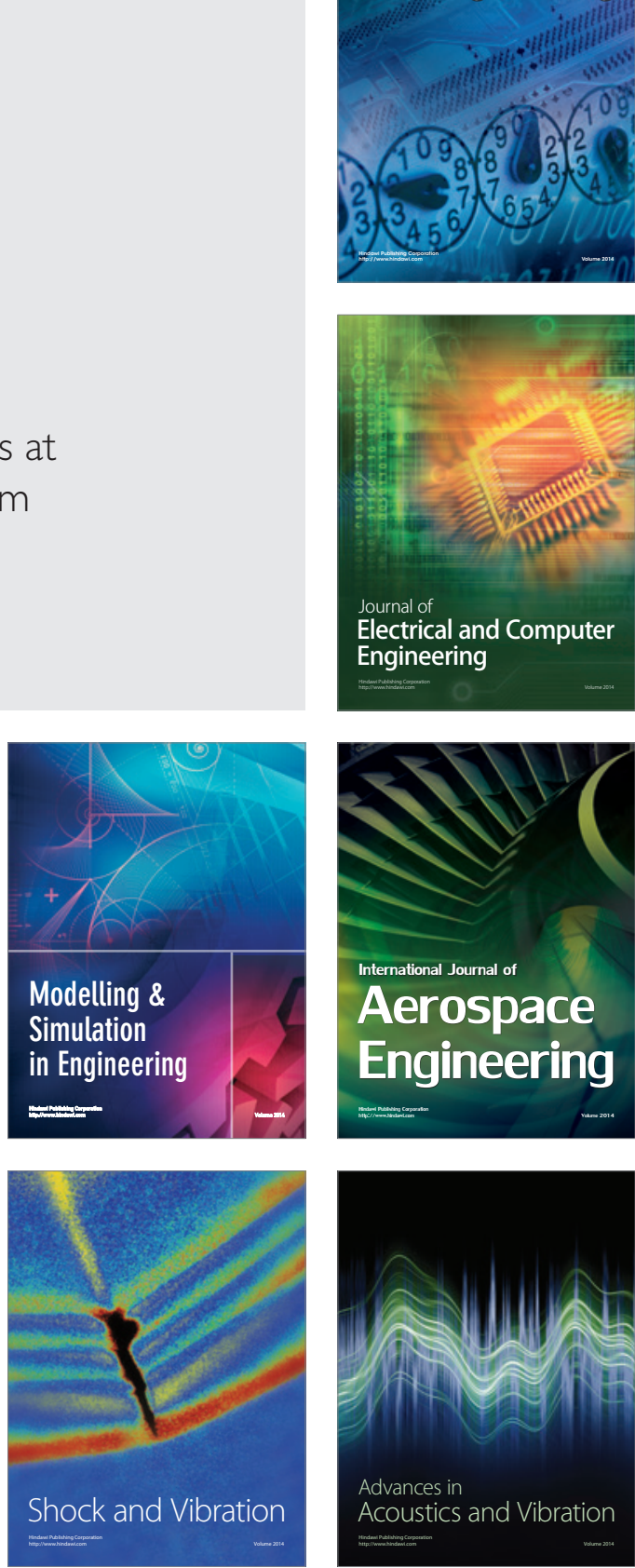